\title{
BMJ Open Latent profile analysis of anxiety disorder among left-behind children in rural Southern China: a cross- sectional study
}

\author{
Haining Liao, ${ }^{1}$ Minyi Pan, ${ }^{1}$ Weinan Li, ${ }^{1}$ Changqi Lin, ${ }^{1}$ Xuhao Zhu, ${ }^{2}$ Xingru Li, ${ }^{3}$ \\ Jinghua Li, ${ }^{3}$ Shudong Zhou ${ }^{1}$
}

To cite: Liao H, Pan M, Li W, et al. Latent profile analysis of anxiety disorder among left-behind children in rural Southern China: a crosssectional study. BMJ Open 2019;9:e029331. doi:10.1136/ bmjopen-2019-029331

- Prepublication history for this paper is available online. To view these files, please visit the journal online (http://dx.doi. org/10.1136/bmjopen-2019029331).

Received 24 January 2019 Revised 29 May 2019 Accepted 5 June 2019

\section{Check for updates}

\section{(c) Author(s) (or their} employer(s)) 2019. Re-use permitted under CC BY-NC. No commercial re-use. See rights and permissions. Published by BMJ.

${ }^{1}$ Guangdong Pharmaceutical University, Guangzhou, China ${ }^{2}$ Qingyuan Center for Disease Control and Prevention, Qingyuan, China

${ }^{3}$ Qingyuan Qingxin District Center for Disease Control and Prevention, Qingyuan, China

Correspondence to Professor Shudong Zhou; zsdong@gdpu.edu.cn

\section{ABSTRACT}

Objectives Previous studies have used latent profile analysis (LPA) to examine rural left-behind children's anxiety. Further study is needed to identify the heterogeneous characteristics of rural left-behind children's anxiety and explore the related factors.

Setting A cross-sectional survey using a school-based sample was conducted in January 2018 in Qingxin district, Qingyuan city, Guangdong province.

Participants 1026 left-behind children (effective response rate of the questionnaire: $95.39 \%$ ).

Main outcome measures Profile latent classes (LC) and anxiety disorder.

Results The LPA identified three anxiety LC: 'Iow anxiety' (56.6\%), 'medium anxiety' (34.8\%) and 'severe anxiety' (8.6\%). The multinomial logistic regression model was used to predict the relationship between personal, family, school factors and anxiety. We found that the variables directly related to lower anxiety classes included age (1214 years), harmonious or fair relationship with classmates, no neglect, harmonious parental relationship and the duration of mother migration $<6$ months.

Conclusions These findings suggested the need for careful consideration of differences in anxieties among rural left-behind children. Identifying latent subgroups may provide an empirical basis for teachers and public health practitioners to implement anxiety intervention efforts.

\section{INTRODUCTION}

Over the past three decades, approximately 280 million Chinese people have migrated from their villages to flourishing cities in search of better employment opportunities which constituted the greatest wave of migration in human history. ${ }^{1}$ Nationwide, about $15 \%$ of all rural families include at least one member who has migrated to the urban areas. $^{2}$ Due to the financial constraints and the school registration system, the children of these migrants cannot live with their parents in cities. Thus, the left-behind children (LBC) of migrant parents are usually looked after by grandparents and extended kin members

\section{Strengths and limitations of this study}

- This study added to the literature by exploring the potential relationship between personal, family, school factors of the left-behind children and their anxiety disorder.

- A latent profile analysis conducted in this study found obvious heterogeneity in the anxiety of left-behind children which may facilitate development of appropriate interventions in a targeted manner.

- The study was limited by its cross-sectional design.

- The participants were restricted to one rural area in southern China, thereby limiting the applicability of the findings to left-behind children in other countries.

- All the participants were recruited from schools, whereby the parental migration may have influenced the children's school attendance which may have resulted in selection bias.

which led to the creation of a special group named as rural 'left-behind children', whose number is rapidly increasing. According to a 2013 report by the All-China Women's Federation, there are 30 million children in China, and more than $10 \%$ of them live in the rural areas without their parents, often in the care of their grandparents or relatives. The report also indicated that Guangdong province has a high concentration of $\mathrm{LBC}$, accounting for $7.18 \%$ of the total number of LBC in China. ${ }^{3}$

As a result of being in a crucial stage of psychological development in childhood and adolescence and with limited interaction with their parents, many of the LBC face developmental and emotional challenges. They may face many negative stimulations in life and easily develop the maladjustment, especially if they are the only children in the family. Such children are more susceptible to psychological problems. ${ }^{45}$ Recent studies have shown that LBC are at a disadvantage in terms of development and social emotional health, 
with higher rates of depression, anxiety, substance abuse and suicide than non-LBC. ${ }^{6-8}$

As the most common type of mental health disorder among children and adolescents anxiety disorder has a prevalence rate of $1.5 \%-21.0 \%$ worldwide, and the anxiety disorder rate among Chinese teenagers is as high as $2.23 \%-14.4 \% .^{9}$ Anxiety is associated with an increased risk of suicidal ideation or attempted suicide in adulthood. ${ }^{9}{ }^{10}$ Studies have shown that the LBC are more likely to have conflicts with peers or teachers than non-LBC, with higher levels of anxiety. Besides, children who are separated from their parents at younger ages have an increased risk of anxiety. ${ }^{112}$ In contrast, other studies have shown that being left-behind does not necessarily lead to children's anxiety, for instance, there were no significant differences in anxiety tendency, trait anxiety and social anxiety between LBC and non-LBC. ${ }^{13}{ }^{14}$ Perhaps the LBC group is a heterogeneous group, resulting in inconsistent findings.

Both latent class analysis (dichotomous outcome) and latent profile analysis (LPA) (continuous outcome) can reduce numerous categorical variables or continuous variables to a few subgroups. ${ }^{15} 16$ Unlike the traditional cluster analysis method, LPA can identify heterogeneous populations through individuals with similar characteristics. Due to these advantages, LPA has been widely used in sociology, psychology and medicine. ${ }^{16}$ For instance, Meng et al conducted an LPA on a sample of 2158 participants and found a best-fitting model with three classes: 'low group anxiety, moderate group anxiety and high group anxiety'. ${ }^{9}$ Fonseca-Pedrero et al using LPA identified four latent classes: 'positive schizotypy', 'low schizotypy' and 'high schizotypy' and their findings can facilitate prevention of psychotic-spectrum disorder and mental health problems. ${ }^{17}$

Currently, there are about 430000 rural LBC in Guangdong province. ${ }^{18}$ In 2015, the Qingyuan women's federation conducted a survey on rural LBC which showed more than 160000 LBC in this city. Qingyuan city is only 2 hours' drive from the Pearl River Delta region which is one of the most developed regions in China. Numerous young and middle-aged labourers go out to work, so Qingyuan is one of the main gathering places of LBC in Guangdong province. Previous studies have examined anxiety among LBC living in the countryside ${ }^{19}$ but few studies have considered the heterogeneity. Thus, in this study, LPA was used to identify homogenous subtypes and influencing factors of anxiety among LBC in Qingyuan city after LPA.

\section{METHODS}

\section{Sample and data collection}

This study had a cross-sectional design. The survey was conducted in January 2018 in Qingxin district, Qingyuan city, Guangdong province. The Qingxin district includes eight villages and towns, of which two were randomly selected. There are 7 junior high schools and 14 primary schools in these two counties. Three middle schools and three primary schools were randomly selected from these 21 schools, representing grades 3-9, yielding 5504 respondents. A total of 5250 participants returned questionnaires (effective response rate: 95.39\%), of which 1026 were $\mathrm{LBC}$ and these were included in the data analysis.

\section{Measures}

Demographic information questionnaire: Participants reported their demographic information (age, gender, grade, type of school, etc), family-related information (parent's education, parent's relationship, parent's migration duration, etc), school-related information (boarding, class committee member, the relationship between classmates, etc).

Rural LBC: In this study, children living in countryside, under 18 years of age, whose both parents or one parent migrated to urban areas at least 6 months ago were referred to as LBC. ${ }^{20}$

Evaluation of neglect: The neglect evaluation scale for primary school students aged 6-11 years in rural areas of China was used to measure neglect of primary school participants. ${ }^{21}$ The neglect evaluation scale for middle school students aged 12-17 years in rural areas of China was used to measure neglect of middle school participants. ${ }^{22}$ The primary school neglect scale contained 58 items and the middle school scale contained 57 items. We used these scales because of the high validity and reliability (Cronbach's $\alpha=0.914$ ). Each item was rated on a 4-point Likert-type format; the positive items were rated from 1 (never) to 4 (always), and the negative items were rated from 4 (always) to 1 (never), and the unanswered the items were rated 0 point. Thus, the total score ranged from 0 to 232 in primary school students and 0-228 in middle school students. The cut-off value of neglect was 140 , and the higher the score, the more neglected the child was.

The Screen for Child Anxiety Related Emotional Disorders (SCARED): Childhood anxiety was measured by SCARED for children with anxiety disorders which was developed by Birmaher in $1977 .{ }^{23}$ It is suitable for children and adolescents with self-assessment anxiety disorders. The screening table for children with anxiety disorder is composed of 41 items, which are divided into five dimensions: somatisation/panic (13 items), generalised anxiety (nine items), separation anxiety (eight items), social phobia (seven items) and school phobia (four items). Total scores ranged from 0 to 83 , with each statement having a rating from 0 to 2 (no such problem $=0$ point; occasional problems $=1$ point; always problems $=2$ points) . The higher the score, the more anxious the child was. SCARED is a reliable and effective anxiety screening tool in Chinese children aged 6-16 years, with high internal consistency $(\alpha=0.43-0.89)$, test-retest coefficients of 0.567 to 0.608 and split reliability of 0.88 . Sensitivity and specificity of diagnosis of anxiety disorder were 0.74 and 0.79 , respectively. ${ }^{24}$ 


\section{Procedure}

The current research was a cross-sectional study. Researchers first communicated with potential primary schools and middle schools for data collection, and those schools that agreed to participate were included in the current study. The participants were rural children, under 18 years of age, whose both parents or one parent had migrated to urban areas at least 6 months ago. All the participants were asked the same questions. At least one parent provided written informed consent and each participant provided oral consent. Next, we asked participants to complete a full set of questionnaires, including the demographic information survey and SCARED. A single-regular survey was conducted in their classroom, which required about $30 \mathrm{~min}$ to complete. No names were collected. Students were not allowed to talk to each other during the survey administration. In order to ensure quality answers and correct understanding of the questions, assistants walked around the classroom to help those who had difficulties. The students were told that there were no right or wrong answers, and they could withdraw from the study at any time.

\section{Statistical analysis}

Pearson correlation coefficients were used to examine the associations between somatisation, generalised anxiety, separation anxiety, social phobia and school phobia. Since most of the coefficients were less than 0.70 , they were suitable as indicator variables.

Next, we conducted an LPA using SCARED subscales to test for existence of discrete groups with similar psychometric profiles. LPA was used to analyse a potential category model of continuous variables. In order to determine the optimal number of classes (ie, class enumeration), we compared each model in LPA. The fit of a 1-class model is initially evaluated, and incrementally increasing models are evaluated until the best class solution is selected. When conducting LPA, there were six model fit indexes available to evaluate the optimal model: Akaike Information Criterion (AIC), Bayesian Information Criterion (BIC), adjusted BIC (aBIC), Lo-Mendell-Rubin (LMR), bootstrapped likelihood ratio test (BLRT) and Entropy. ${ }^{25}$ Entropy evaluates the quality of each class resulting from LPA, with a priority value of more than 0.80 and close to 1.0 indicating much clearer results. ${ }^{26}{ }^{27}$ The estimated model was compared with a model with $k-1$ class or classes using LMR and BLRT, where $k$ was equal to the number of classes. In the LMR and BLRT, a low value and statistically significant $\mathrm{p}$ value indicated that the estimated model is better than the model with one less class. The AIC, BIC and aBIC are usually used to compare different counterpart models, with the lowest value of each indicator indicating a best-fitting model..$^{28}$

Third, after determining the best latent class solution, we compared the somatisation, generalised anxiety, separation anxiety, social phobia and school phobia scores among the latent class groups by using the analysis of covariance (ANOVA). Then we conducted rank-sum tests to compare the demographic, family and school information of LBC among the latent classes. Finally, multinomial logistic regression analysis was conducted to examine the associations between the latent classes and LBC anxiety. Control variables included gender, grade, classmate relationship, no neglect, parental emotion, the duration of father's migration, the duration of mother migration and so on. An OR greater than 1.00 suggested that the predictor was correlated with increasing anxiety.

The LPA was conducted with the Mplus V.7.0. Descriptive statistics and multinomial logistic regression analyses were performed with SAS V.9.4. All tests were two-sided and $\alpha<0.05$ was considered as statistically significant.

\section{Patient and public involvement}

The study was designed to explore the LBC' s anxiety. The schools randomly selected all participated in the survey. However, the LBC who participated did so anonymously, and therefore the study team was unable to disseminate the results to study participants. But we had shared the results of the analysis with the participating schools. Based on the results of the survey, we recommended relevant interventions.

\section{RESULTS}

One to four classes were tested by LPA. As the number of classes increased, the BIC and ABIC values decreased. The 1-class and 2-class models were first compared. The LMR-A $p$ value of the 2-class model reached statistically significance, suggesting that the 2-class model was better than the 1-class model. Then, compared with the 3-class and 4-class profile models, the 3-class and 4-class models of the LMR-A p value did not reach statistical significance but 3-class showed a lower AIC, BIC and aBIC than 2-class. As compared with 3-class, the indexes of the 4-class were only slightly decreased $(<300)$. The 3-class model had higher entropy than the 4-class model. Therefore, to better represent the data, we selected the 3-class as the best solution. Among the 3-class solution, class 1 described $56.6 \%$ and comprised 581 LBC, class 2 described $34.8 \%$ and comprised $357 \mathrm{LBC}$, and class 3 described $8.6 \%$ and comprised 88 LBC (tables 1 and 2).

As shown in figure 1, with lowest scores on somatisation, generalised anxiety, separation anxiety, social phobia and school phobia, class 1 was named as low-anxiety group. Class 2 was named as medium-anxiety group because all anxiety domain scores were between class1 and class 3 . Class 3 showed high scores on all anxiety domains and was named as severe-anxiety group.

An ANOVA was conducted for validating the anxiety latent profiles. Table 3 shows the results of ANOVA and multiple comparisons. We found three effect sizes: low, medium and severe. The three latent profiles revealed different patterns of correlations with somatisation, generalised anxiety, separation anxiety, social phobia and school phobia. Particularly, as compared with other two latent classes, the 'severe-anxiety group' demonstrated 
higher mean scores. The low anxiety type of LBC in five dimensions of anxiety score was significantly lower than medium anxiety type and severe anxiety type of LBC, medium anxiety type of LBC in five dimensions of anxiety score was significantly higher than that of low anxiety type of LBC, significantly lower than severe anxiety type of LBC. The results showed that the latent profile classes of LBC anxiety can accurately identify the degree of LBC anxiety which also indicates that this potential classification is effective.

The ages of these respondents ranged from 8 to 17 years, with a mean age of $13.17 \pm 1.94$ years; $12-14$ years old children had higher prevalence rates of anxiety. Overall, the proportion with severe anxiety was similar between boys and girls. There were significant differences in the proportion health status among three latent classes $(p<0.05)$. The LBC with severe anxiety had higher prevalence rates of neglect. However, there were no significant differences among the three group in terms of only child and parent education level $(\mathrm{p}>0.05)$ (table 4$)$.

In the severe anxiety class, the LBC who had poor/fair emotions between their parents, parental divorce and unharmonious relationship with classmates accounted for a greater proportion $(40.91 \%, 12.94 \%$ and $13.64 \%$, respectively) than the other two classes. When stratified by parent's migration status, LBC whose mothers migrated over 6 months ago had higher anxiety disorder (56.82\%). There were no significant differences in the duration of father's migration, board, class committee member and class teacher's attitude among the three classes $(p>0.05)$ (table 5).

To identify the relevant factors of anxiety disorder among the three classes, multinomial logistic regression analysis was conducted. When the other covariates remained constant, LBC aged 12-14 years were more likely to belong to the low-anxiety group (adjusted OR $(\mathrm{AOR})=2.19,95 \% \mathrm{CI}: 1.02$ to 4.69$)$ and medium-anxiety group (AOR=4.21, 95\% CI: 1.91 to 9.31). As compared with unharmonious relationship with classmates, LBC with harmonious relationship with classmates were more likely to belong to the low-anxiety group $(\mathrm{AOR}=4.52$; 95\% CI: 1.88 to 10.84 ) and medium-anxiety group $(\mathrm{AOR}=3.51 ; 95 \% \mathrm{CI}: 1.41$ to 8.77$)$ rather than the severe-anxiety group. LBC with fair relationship with classmates were more likely to belong to the low-anxiety group $(\mathrm{AOR}=4.53 ; 95 \% \mathrm{CI}: 1.86$ to 11.06$)$ and medium-anxiety group (AOR=2.98; $95 \% \mathrm{CI}$ : 1.17 to 7.64 ) rather than the severe-anxiety group. The non-neglected LBC were associated with a slightly increased likelihood of belonging to the low-anxiety group than severe-anxiety group (AOR $=1.94 ; 95 \%$ CI: 1.20 to 3.14). Moreover, the LBC with harmonious parental marital relations were more likely to belong to the low-anxiety group than severe-anxiety group (AOR=2.94; $95 \% \mathrm{CI}: 1.74$ to 4.96$)$. The study further indicated that the LBC whose mothers migrated less than 6 months ago were more likely to belong to the low-anxiety group (AOR=1.67; 95\% CI: 1.03 to 2.71$)$ and the medium-anxiety group (AOR=1.77;95\% CI: 1.07 to 
Table 2 Average latent profile class probabilities for the most likely class membership (row) by latent class (column)

\begin{tabular}{lllll}
\hline \multirow{2}{*}{ Latent class } & \multicolumn{2}{l}{ Class membership } & \\
\cline { 2 - 5 } & $\mathbf{1}(\mathbf{n}=\mathbf{5 8 1})$ & $\mathbf{2}(\mathbf{n}=\mathbf{3 5 7})$ & $\mathbf{3}(\mathbf{n = 8 8})$ & M (SD) total score for SCARED \\
\hline 1 & 0.95 & 0.05 & 0.00 & $10.66 \pm 5.54$ \\
2 & 0.07 & 0.90 & 0.03 & $26.86 \pm 5.45$ \\
3 & 0.00 & 0.08 & 0.92 & $43.43 \pm 7.08$ \\
\hline
\end{tabular}

The columns refer to the latent class, and the rows refer to the most likely profit membership.

SCARED, Screen of Child Anxiety Related Emotional Disorders.

2.91) than in the severe-anxiety group. Latent profile memberships were not associated with gender, grade, health status and parent's divorce (table 6).

\section{DISCUSSION}

The current study was designed to explore the latent profiles of LBC anxiety and found three latent profile classes. Based on the scoring pattern of SCARED sub-scales, they were divided into low-anxiety group, medium-anxiety group and severe-anxiety group, respectively.

The low-anxiety group consisted of $56.6 \%$ of participants. The characteristics were low levels of all anxiety themes: somatisation, generalised anxiety, separation anxiety, social phobia and school phobia. This indicated that low anxiety type LBC can better adjust and cope with their parents' migration. Some studies have shown that some LBC did not have psychological and behavioural problems after separation from their parents. ${ }^{29}{ }^{30}$ Migration may positively affect the educational opportunities of children because their parents are aware of the significance of school and encourage their children to receive a high level of education. ${ }^{431} \mathrm{~A}$ high level of education can enhance their ability to cope with stress. Although the overall ratings of anxiety in the low-anxiety group were low, the social phobia subscale demonstrated the highest score and the school phobia had the lowest score, respectively. Hence, schools should create more opportunities for the LBC to communicate and participate in group activities.

The second group, named the medium-anxiety group, was composed of $34.8 \%$ of the population. Generalised anxiety and social phobia received higher scores in this

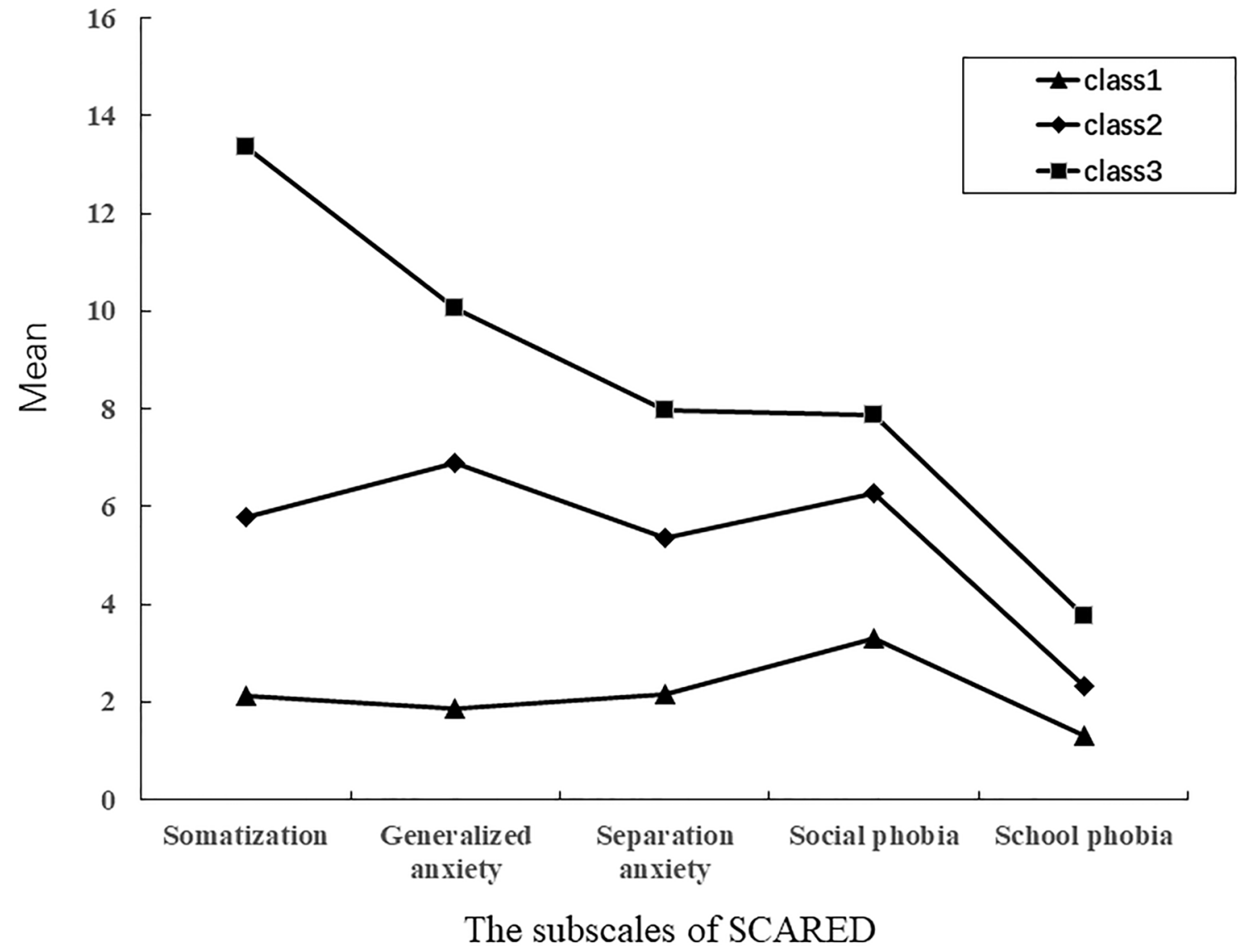

Figure 1 Three profiles of anxiety of the best-fitting three-class pattern. 
Table 3 Mean comparisons across three latent classes

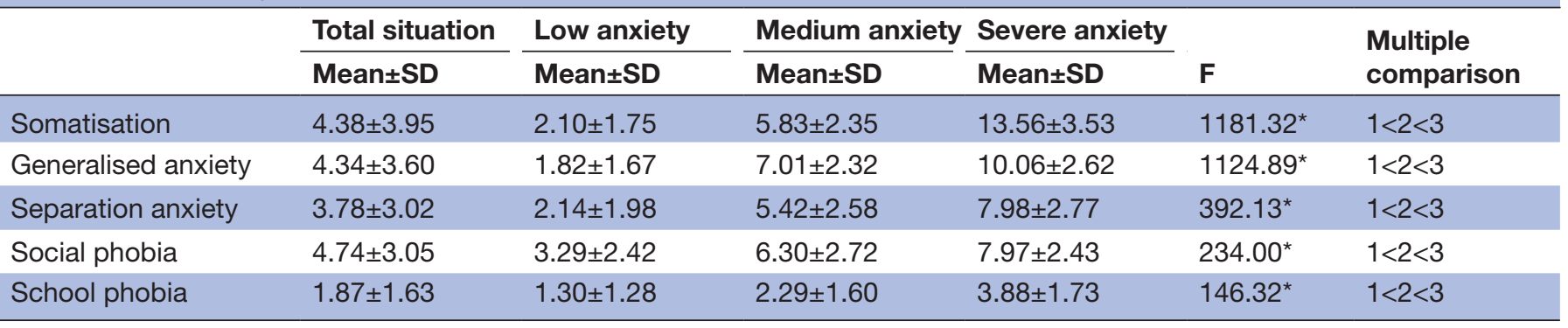

${ }^{*} \mathrm{P}<0.001$.

group than the other anxiety manifestations. Although the average score of SCARED in the medium-anxiety group was higher than the clinical cut-off point for scale (ie, 23), ${ }^{24}$ it was lower compared with the mean score of clinical anxiety adolescents (34.79). ${ }^{9}$ Previous studies demonstrated that generalised anxiety became the main anxiety in the LBC. ${ }^{32}$ This may be related to their growth environment. Their parents worked away from home so the children could not receive effective parental support. Additionally, the LBC are exposed to examination-oriented education and worry about the future, thereby resulting in more generalised anxiety.

The third group, named the severe-anxiety group, consisted of $8.6 \%$ of the participants. Their mean scores were much higher than the cut-off score of SCARED. ${ }^{24}$ The participants were characterised by high levels of all anxiety themes: somatisation, generalised anxiety, separation anxiety, social phobia and school phobia. Compared with the other anxiety manifestations, somatisation demonstrated higher score. This finding would help us to focus on relieving the LBC somatisation symptoms, such as abdominal pain, dizziness, fatigue and so on. In addition to actively addressing the acute physical symptoms of children, schools and families should also strengthen communication and carry out psychological counselling and psychotherapy. ${ }^{33}$

In this study, LBC anxiety level differed with age which was consistent with previous studies. ${ }^{34}$ It indicated that parents' migration had an important influence on the mood of younger children. When the children are young, parents are their guides for growth. When facing difficulties, children generally receive parental help and

Table 4 Demographic information for three profile latent classes among different left-behind children

\begin{tabular}{|c|c|c|c|c|c|}
\hline Variable & Low anxiety & Medium anxiety & Severe anxiety & $\chi^{2}$ & $P$ value \\
\hline Age (years) & & & & 16.62 & 0.0002 \\
\hline $8-11$ & $158(27.19)$ & 55 (15.41) & 32 (36.36) & & \\
\hline $12-14$ & $263(45.27)$ & $182(50.98)$ & 32 (36.36) & & \\
\hline $15-17$ & $160(27.54)$ & $120(33.61)$ & $24(27.27)$ & & \\
\hline Gender & & & & 14.28 & 0.0008 \\
\hline Male & $313(53.87)$ & $147(41.18)$ & $44(50.00)$ & & \\
\hline Female & $268(46.13)$ & $210(58.82)$ & $44(50.00)$ & & \\
\hline Grade & & & & 11.04 & 0.0040 \\
\hline Primary & 290 (49.91) & 139 (38.94) & $43(48.86)$ & & \\
\hline Middle & 291 (50.09) & $218(61.06)$ & 45 (51.14) & & \\
\hline Only child & & & & 5.31 & 0.0702 \\
\hline No & $456(78.49)$ & 302 (84.59) & 71 (80.68) & & \\
\hline Yes & 125 (21.51) & 55 (15.41) & 17 (19.32) & & \\
\hline Health status & & & & 11.74 & 0.0028 \\
\hline Fair/poor & $121(20.83)$ & $102(28.57)$ & 30 (34.09) & & \\
\hline Good & $460(79.17)$ & 255 (71.43) & 58 (65.91) & & \\
\hline Boarding & & & & 5.59 & 0.0611 \\
\hline No & $365(62.82)$ & $206(57.70)$ & 45 (51.14) & & \\
\hline Yes & $216(37.18)$ & $151(42.30)$ & $43(48.86)$ & & \\
\hline Total & 581 (56.63) & $357(34.80)$ & 88 (8.57) & 1026 & \\
\hline
\end{tabular}


Table 5 Family and school information for three profile latent classes among different left-behind children

\begin{tabular}{|c|c|c|c|c|c|}
\hline Variable & Low anxiety & Medium anxiety & Severe anxiety & $\chi^{2}$ & $P$ value \\
\hline Mother's education & & & & 4.53 & 0.1036 \\
\hline Primary school or below & $192(33.05)$ & 135 (37.82) & $28(31.82)$ & & \\
\hline Junior middle school & $229(39.41)$ & $142(39.78)$ & $33(37.50)$ & & \\
\hline High school and above & $71(12.22)$ & $40(11.20)$ & $15(17.05)$ & & \\
\hline Unknown & 89 (15.32) & $40(11.20)$ & $12(13.64)$ & & \\
\hline Father's education & & & & 2.23 & 0.3275 \\
\hline Primary school or below & $144(24.78)$ & $87(24.37)$ & $21(23.86)$ & & \\
\hline Junior middle school & $282(48.54)$ & $188(52.66)$ & $36(40.91)$ & & \\
\hline High school and above & $68(11.70)$ & $43(12.04)$ & 17 (19.32) & & \\
\hline Unknown & 87 (14.97) & 39 (10.92) & $14(15.91)$ & & \\
\hline Parental relationship & & & & 33.98 & $<0.0001$ \\
\hline Harmonious & $481(82.79)$ & $253(70.87)$ & 52 (59.09) & & \\
\hline Fair/poor & $100(17.21)$ & $104(29.13)$ & $36(40.91)$ & & \\
\hline The duration of father migration & & & & 2.65 & 0.2663 \\
\hline Within half a year & $325(55.94)$ & $187(52.38)$ & $42(47.73)$ & & \\
\hline Over half a year & $256(44.06)$ & $170(47.62)$ & $46(52.27)$ & & \\
\hline The duration of mother migration & & & & 7.49 & 0.0237 \\
\hline Within half a year & $341(58.69)$ & $201(56.30)$ & $38(43.18)$ & & \\
\hline Over half a year & 240 (41.31) & $156(43.70)$ & $50(56.82)$ & & \\
\hline \multicolumn{6}{|l|}{ Parental divorce ${ }^{*}$} \\
\hline No & $533(93.02)$ & 307 (88.22) & $74(87.06)$ & 7.61 & 0.0223 \\
\hline Yes & $40(6.98)$ & $41(11.78)$ & $11(12.94)$ & & \\
\hline Neglected & & & & 13.05 & 0.0015 \\
\hline No & $401(69.02)$ & $221(61.90)$ & $45(51.14)$ & & \\
\hline Yes & $180(30.98)$ & $136(38.10)$ & $43(48.86)$ & & \\
\hline Class committee member & & & & 1.46 & 0.4824 \\
\hline No & 375 (64.54) & 219 (61.34) & $59(67.05)$ & & \\
\hline Yes & $206(35.46)$ & $138(38.66)$ & 29 (32.95) & & \\
\hline Classmate relationship & & & & 9.38 & 0.0092 \\
\hline Harmonious & $363(62.48)$ & 239 (66.95) & $46(52.27)$ & & \\
\hline Fair & $201(34.60)$ & $105(29.41)$ & 30 (34.09) & & \\
\hline Unharmonious & 17 (2.93) & $13(3.64)$ & $12(13.64)$ & & \\
\hline Class teacher's attitude & & & & 4.82 & 0.0897 \\
\hline Kindness/fair & $405(69.71)$ & 257 (71.99) & $54(61.36)$ & & \\
\hline Strict & $155(26.68)$ & $96(26.89)$ & 28 (31.82) & & \\
\hline Extreme attitudes & $21(3.61)$ & $4(1.12)$ & $6(6.82)$ & & \\
\hline
\end{tabular}

*Missing 20 persons.

support, without which they are susceptible to negative emotions. After puberty, peer relations gradually replace the parent-child relationship. When they encounter external pressures or adverse events, children are more likely to use self-help or seek support from classmates and friends. Some recent studies have shown that close peer relationships (ie, friendship) were crucial for the well-being of LBC in rural China. A strong friendship can decrease the loneliness, depression and anxiety of LBC. ${ }^{2} 3536$ In addition, previous studies indicate that students who received less support from close peers are expected to develop increased anxiety symptoms. ${ }^{3738}$

Given the influence of family factors, our study found that neglected LBC are more prone to anxiety which was similar to the results of a previous study. ${ }^{4}$ Mothers are primary caregivers for children in our traditional families. If both parents migrate, the LBC are usually looked after by grandparents or other relatives. The caregivers 
Table 6 Multinomial logistic regressions for predicting in three profile latent classes among the left-behind children (AOR value)

\begin{tabular}{|c|c|c|c|c|c|c|}
\hline \multirow[b]{2}{*}{ Variable } & \multicolumn{2}{|c|}{ Low anxiety } & \multirow[b]{2}{*}{$P$ value } & \multicolumn{2}{|c|}{ Medium anxiety } & \multirow[b]{2}{*}{$P$ value } \\
\hline & AOR & $\mathrm{Cl}(95 \%)$ & & AOR & $\mathrm{Cl}(95 \%)$ & \\
\hline \multicolumn{7}{|l|}{ Age (years) } \\
\hline $8-11$ & 1.00 & & & 1.00 & & \\
\hline $12-14$ & 2.19 & 1.02 to 4.69 & 0.0433 & 4.21 & 1.91 to 9.31 & 0.0004 \\
\hline $15-17$ & 2.36 & 0.86 to 6.46 & 0.0956 & 4.43 & 1.57 to 12.50 & 0.0049 \\
\hline \multicolumn{7}{|l|}{ Gender } \\
\hline Male & 1.00 & & & 1.00 & & \\
\hline Female & 0.82 & 0.51 to 1.33 & 0.4282 & 1.44 & 0.88 to 2.36 & 0.1475 \\
\hline \multicolumn{7}{|l|}{ Grade } \\
\hline Primary & 1.00 & & & 1.00 & & \\
\hline Middle & 0.61 & 0.27 to 1.37 & 0.2311 & 0.66 & 0.29 to 1.50 & 0.3214 \\
\hline \multicolumn{7}{|l|}{ Health status } \\
\hline Fair/poor & 1.00 & & & 1.00 & & \\
\hline Good & 0.63 & 0.38 to 1.06 & 0.0821 & 0.91 & 0.53 to 1.54 & 0.8407 \\
\hline \multicolumn{7}{|c|}{ Classmate relationship } \\
\hline Unharmonious & 1.00 & & & 1.00 & & \\
\hline Harmonious & 4.52 & 1.88 to 10.84 & 0.0007 & 3.51 & 1.41 to 8.77 & 0.0071 \\
\hline Fair & 4.53 & 1.86 to 11.06 & 0.0009 & 2.98 & 1.17 to 7.64 & 0.0227 \\
\hline \multicolumn{7}{|l|}{ Neglected } \\
\hline Yes & 1.00 & & & 1.00 & & \\
\hline No & 1.94 & 1.20 to 3.14 & 0.0072 & 1.42 & 0.86 to 2.33 & 0.1692 \\
\hline \multicolumn{7}{|l|}{ Parental relationship } \\
\hline Fair/poor & 1.00 & & & 1.00 & & \\
\hline Harmonious & 2.94 & 1.74 to 4.96 & $<0.0001$ & 1.67 & 0.98 to 2.85 & 0.0609 \\
\hline \multicolumn{7}{|c|}{ Duration of mother migration } \\
\hline Over half a year & 1.00 & & & 1.00 & & \\
\hline Within half a year & 1.67 & 1.03 to 2.71 & 0.0384 & 1.77 & 1.07 to 2.91 & 0.0264 \\
\hline \multicolumn{7}{|l|}{ Parental divorce } \\
\hline Yes & 1.00 & & & 1.00 & & \\
\hline No & 1.18 & 0.54 to 2.56 & 0.0821 & 0.92 & 0.43 to 2.01 & 0.7181 \\
\hline
\end{tabular}

Severe anxiety was a control group.

AOR, adjusted OR.

cannot communicate well in time which makes the LBC more vulnerable to neglect and anxiety. ${ }^{39} 40$

Harmonious parental relationship can reduce the risk of anxiety. According to previous literature, problematic parental relationships constitute additive risk factors that directly and independently foster anxiety symptoms, especially interparental conflicts. ${ }^{41}{ }^{42}$ Perhaps the parents spend most of their time to solve their marital discord. Hence, little attention is paid to their children's lives, emotional and behavioural problems. Our study also found that the separation of children from their mothers had a significantly higher impact on children's emotional disorders than that of fathers. It may be that emotional communication with mothers can significantly alleviate children's depression/anxiety and other adverse emotional reactions. This was similar to the findings of Shen Min $e$ al. ${ }^{6}$

The results from this study have clinical implications for the intervention of LBC in Qingyuan city. In the sub-group of LBC with low anxiety, targeted prevention and intervention for social phobia symptoms may be beneficial. Similarly, for LBC with medium anxiety, prevention and intervention efforts may target generalised anxiety and social phobia symptoms. However, the LBC who are seriously anxious may require intervention in all aspects. From the factors influencing anxiety of LBC, the potential anxiety of this population should be noticed by parents, teachers and other individuals. At 
least one of the parents, particularly the mother, should live with their children because they play a significant role in developing their children's sense of security and social skills. ${ }^{6}$ Moreover, parents should create a harmonious family atmosphere at home and avoid quarrelling in front of their children. As for schools, psychological education lectures should be regularly held, and teachers should pay close attention to children's emotions and communicate with them more. In addition, parents or teachers should also guide the LBC to establish positive relationship with their peers and ease their anxiety. Last but not the least, the government should further improve relevant policies (education, medical care, residence, etc) to promote the physical and mental health of LBC.

The analysis of anxiety domains in this study revealed no significant relationship with gender, school type, health status and parent's divorce among LBC. Some previous studies indicated that gender had no significant difference in childhood anxiety disorder. ${ }^{30}{ }^{43}$ In contrast, other studies suggested that female students and middle school students reported greater levels of anxiety than their male counterparts and primary school students. ${ }^{6}$ Similarly, some studies found that parent's divorce was not the decisive factor affecting the psychological development of children and adolescents. ${ }^{44}$ However, other studies showed that parent's divorce affected the child's mental state, leading to higher frequency of depression, violence, social problems and high risk for suicidal attempts. ${ }^{45}$ Direct comparisons cannot be made among these studies because they did not use the same sampling strategy or have all the same measures. The relationship between gender, grade, parent's divorce and anxiety symptoms needs further exploration.

This study had some limitations. First, this study could not determine the direction of causality between related factors and anxiety because of its cross-sectional design. Second, all the participants were recruited from schools, so the parental migration may have influenced the children's school attendance which may have resulted in selection bias. Third, although we made efforts to ensure the representativeness of the sample, the participants were a convenience sample from one rural county in southern China, thereby limiting the ability to extend the findings to LBC in other countries. Last, more variables related to anxiety disorder should be considered in future research since many related factors (ie, household economic status, caregivers, etc) may influence LBC anxiety.

\section{CONCLUSIONS}

Despite the potential limitations, this study found obvious heterogeneity in the anxiety of LBC in Qingyuan city which could be divided into three latent profile classes: 'low-anxiety group', 'medium-anxiety group' and 'severe-anxiety group'. LBC who were younger, had a poor relationship with their classmates, who were neglected, whose parents had marital discord and whose mothers were away for more than 6 months were more anxious.
Acknowledgements The authors thank the Qingyuan City Center for Disease Control and Prevention Guangdong, China.

Contributors $\mathrm{HL}$ planned the statistical analysis and wrote and revised the manuscript. MP designed the project. MP, HL, WL, CL, XZ, XL and JL collected, managed the data. SZ provided a critical review and thoroughly edited the manuscript and check the English language grammar.

Funding This work was supported by the self-financing science and technology plan project for social development in Qingyuan City grant number [2017B072].

Competing interests None declared.

Patient consent for publication Not required.

Ethics approval Ethics Committee of Qingyuan Center for Disease Control and Prevention.

Provenance and peer review Not commissioned; externally peer reviewed.

Data sharing statement Data are available upon reasonable request.

Open access This is an open access article distributed in accordance with the Creative Commons Attribution Non Commercial (CC BY-NC 4.0) license, which permits others to distribute, remix, adapt, build upon this work non-commercially, and license their derivative works on different terms, provided the original work is properly cited, appropriate credit is given, any changes made indicated, and the use is non-commercial. See: http://creativecommons.org/licenses/by-nc/4.0/.

\section{REFERENCES}

1. Zhang L. The Orphans of China's Economic Miracle. Secondary The Orphans of China's Economic Miracle. 2018. https://www.nytimes. com/2018/03/27/opinion/china-left-behind-children.html

2. Wen M, Lin D. Child development in rural China: children left behind by their migrant parents and children of nonmigrant families. Child Dev 2012;83:120-36.

3. Wang R, Yang DF. Pay attention to left-behind children and migrant children. Trade Union Inform 2013:50.

4. Chen M, Chan KL. Parental absence, child victimization, and psychological well-being in rural China. Child Abuse Negl 2016;59:45-54.

5. Guan H, Tao XW, Fan ZY, et al. O-207 The Status Of Early Childhood Development Of "left-behind Children": A Cross-sectional Survey In Rural China. Arch Dis Child 2014;99(Suppl 2):A104.1-A104.

6. Shen M, Gao J, Liang Z, et al. Parental migration patterns and risk of depression and anxiety disorder among rural children aged 10-18 years in China: a cross-sectional study. BMJ Open 2015;5:e007802.

7. Su S, Li X, Lin D, et al. Psychological adjustment among left-behind children in rural China: the role of parental migration and parent-child communication. Child Care Health Dev 2013;39:162-70.

8. Tan M, Chen M, Li J, et al. Depressive symptoms and associated factors among left-behind children in China: a cross-sectional study. BMC Public Health 2018;18:1059-66.

9. Yu M, Chasson GS, Wang M, et al. The latent profile analysis of Chinese adolescents' anxiety: Examination and validation. $J$ Anxiety Disord 2018;59:74-81.

10. Cheng J, Sun YH. Depression and anxiety among left-behind children in China: a systematic review. Child Care Health Dev 2015;41:515-23.

11. Valtolina GG, Colombo C. Psychological well-being, family relations, and developmental issues of children left behind. Psychol Rep 2012;111:905-28.

12. Wei $\mathrm{S}$, Cheng J, Wang $\mathrm{YC}$, et al. Anxiety and its influencing factors among rural left-behind preschoolers in Chizhou City,2015. Pract Prevent Med 2016;23:1326-9.

13. Jin YL, Xj W, Zhang XB. Influencing factors of mental health among left-behind children in rural area. Chin J Public Health 2010;26:1224-5.

14. Zhu Y, Hu J, Yj Y, et al. Mental health and coping styles among rural left-behind children. Chin J School Health 2014;35:1657-9.

15. Cella M, Serra M, Lai A, et al. Schizotypal traits in adolescents: links to family history of psychosis and psychological distress. Eur Psychiatry 2013;28:247-53.

16. Wang M, Hanges PJ. Latent Class Procedures: Applications to Organizational Research. Organ Res Methods 2011;14:24-31.

17. Fonseca-Pedrero E, Ortuno-Sierra J, Muniz J, et al. Latent profile analysis of psychosis liability in a community-derived sample of adolescents: Links with mental health difficulties, suicidal ideation, bipolar-like experiences and psychotic-like experiences. Early Interv Psychiatry 2018:1-10. 
18. Duan CG, Ld L, Guo J, et al. The Basic Status of Survival and Development of Left-behind Children in Rural Areas in China. Pop J 2013;35:37-49.

19. Lin XJ, Liu K, Sz H, et al. Psychological health status among left-behind children in underdevelopmented areas of Guangdong province. Chin J Child Health Care 2015;23:295-7.

20. Huang Y, Zhong XN, Li QY, et al. Health-related quality of life of the rural-China left-behind children or adolescents and influential factors: a cross-sectional study. Health Qual Life Outcomes 2015;13:29.

21. Pan J, Yang W, Chen J, et al. [Development of Neglect Evaluation Scale for primary school students aged 6-11 years old in rural areas of China]. Zhonghua Yu Fang Yi Xue Za Zhi 2014;48:476-83.

22. Pan JP, Yang WY, Chen JQ. Development of neglect evaluation norms for 12-17 middle school students in rural areas of China. Chin J School Health 2014;35:165-8.

23. Birmaher B, Khetarpal S, Brent D, et al. The Screen for Child Anxiety Related Emotional Disorders (SCARED): scale construction and psychometric characteristics. J Am Acad Child Adolesc Psychiatry 1997;36:545-53.

24. Wang K. Chinese urban norm for screening anxiety-related emotional disorders in children. Chin J Clin Rehabil 2005;9:254-6.

25. Netherlands S. Latent Class Cluster Analysis. 2014;34:3508-08.

26. Helmers BR, Jacques-Tiura AJ, Abbey A. Associations between young adult men's patterns of alcohol use and sexual behavior: A latent profile analysis of two independent samples. Addict Behav 2019;90:20-6.

27. Chirinos DA, Murdock KW, LeRoy AS, et al. Depressive symptom profiles, cardio-metabolic risk and inflammation: Results from the MIDUS study. Psychoneuroendocrinology 2017;82:17-25.

28. Fonseca-Pedrero E, Ortuño-Sierra J, de Álbeniz AP, et al. A latent profile analysis of schizotypal dimensions: Associations with psychopathology and personality. Psychiatry Res 2017;253:110-5.

29. Jiang $\mathrm{YH}$, Sun $\mathrm{YH}$, Yang LS, et al. Psychological resilience and its influencing factors of left-behind children in a rural area of Anhui Province. Chin J School Health 2013;34:365-7.

30. Gracia F, Kelly RC, Zhao CY. Heath impacts of parental migration on left-behind children and adolescents: a systematioc review and meta-analysis. The Lancet 2018;392:2567-82.

31. Sun X, Tian Y, Zhang Y, et al. Psychological development and educational problems of left-behind children in rural China. Sch Psychol Int 2015;36:227-52.
32. Fan F, Ly S, Su Y, et al. Anxiety Structure by Gender and Age Group in a Chinese Children Sample of 12 Cities. Chin Ment Health J 2008;22:241-5.

33. Rockhill C, Kodish I, DiBattisto C, et al. Anxiety disorders in children and adolescents. Curr Probl Pediatr Adolesc Health Care 2010;40:66-99.

34. Yq H, Zhu CY. Comparative Study on Mental Health Status of Leftbehind Children in Rural Areas at Different School Ages. Soci Sci Hunan 2015;01:105-10.

35. Zhao J, Liu X, Wang M. Parent-child cohesion, friend companionship and left-behind children's emotional adaptation in rural China. Child Abuse Negl 2015;48:190-9.

36. Wang XL, Xy H, Shen JL. Affection of Left Children's Friendship Quality on Lonelines and Depression. Chin J Clin Psychol 2011;19:252-4.

37. Gazelle H. Anxious solitude/withdrawal and anxiety disorders: conceptualization, co-occurrence, and peer processes leading toward and away from disorder in childhood. New Dir Child Adolesc Dev 2010;2010:67-78.

38. Motoca LM, Williams S, Silverman WK. Social skills as a mediator between anxiety symptoms and peer interactions among children and adolescents. J Clin Child Adolesc Psychol 2012;41:329-36.

39. Guo J, Ren X, Wang X, et al. Depression among Migrant and LeftBehind Children in China in Relation to the Quality of Parent-Child and Teacher-Child Relationships. PLoS One 2015;10:e0145606.

40. Zhao C, Wang F, Zhou X, et al. Impact of parental migration on psychosocial well-being of children left behind: a qualitative study in rural China. Int J Equity Health 2018;17:80-9.

41. Yj Y, Ao Y, Shi SF. Life event and family influential factors among different rural left-behind children. Chin J Public Health 2013;29:339-42.

42. Cummings EM, Koss KJ, Davies PT. Prospective relations between family conflict and adolescent maladjustment: security in the family system as a mediating process. J Abnorm Child Psychol 2015;43:503-15

43. $\mathrm{Cz}$ H, Feng YT, QI L. Analysis on Anxiety Emotion Among the Leftbehind Children in Ningxia. Med Innov Chin 2013;10:1-4.

44. Deng LY, Zhao XY, Fang XY. The Influence of Parental Divorce on Children and Adolescents' Mental Development: The Important Role of Inter-Parental Conflict. Psychol Dev Edu 2016;32:246-56.

45. Haimi M, Lerner A. The Impact of Parental Separation and Divorce on the Health Status of Children, and the Ways to Improve it. J Clin Med Genomics 2016;4:1-7. 